\title{
La dimensión de lo barrial en la encrucijada de la comunicación, la ciudad y el espacio público. Una mirada etnográfica sobre la subjetivación urbana contemporánea
}

\author{
Eduardo Álvarez Pedrosian \\ Universidad de la República, Uruguay \\ eduardo.alvarez@fic.edu.uy
}

Recibido: 27/07/2018 / Aceptado: 26/09/2018

doi: 10.26439/contratexto2018.n030.3149

\begin{abstract}
Resumen. Planteamos la dimensión de lo barrial como llave para la comprensión de los fenómenos urbanos en relación con las prácticas de sus habitantes, procurando a un mismo tiempo dar lugar a la heterogeneidad y la conexión de perspectivas y experiencias. En la introducción se esbozan los alcances de la propuesta, su relación con las temáticas de la comunicación, la ciudad y el espacio público. Luego, nos centramos en la comunicación urbana en tanto universo de las mediaciones que inevitablemente expresa y con las que produce subjetividad, así como en casos etnográficos en los que pueden observarse dinámicas que son tanto intencionales como involuntarias en sus efectos. Posteriormente, problematizamos la distinción entre lo barrial y lo urbano, también desde casos etnográficos, para afirmar una perspectiva compositiva de los territorios según la multiplicidad de elementos y sus escalas relativas. Por último, concluimos retomando la problematización del espacio público y formulando consideraciones más amplias sobre esferas similares en otros sentidos y prácticas.
\end{abstract}

Palabras clave: barrio / ciudad / comunicación / espacio público / subjetividad 


\title{
The neighborhood dimension at the crossroads of communication, city and public space. An ethnographic view of the contemporary urban subjectivation
}

\begin{abstract}
АвsтRAст. We propose the neighborhood dimension as a key for understanding urban phenomena in relation to its inhabitants' practices, trying at the same time to give rise to the heterogeneity and connection of perspectives and experiences. In the introduction, we delineate the proposal's scope, its relationship with communication, the city and the public space. Then, we focus on urban communication as a universe of mediations that inevitably express and thus produce subjectivity, and on ethnographic cases where intentional and involuntary dynamics can be observed in their effects. Later, we problematize the distinction between neighborhood and urban area, also based on ethnographic cases, to affirm a compositional perspective of the territories, according to the multiplicity of elements and their relative scales. Finally, we conclude by taking up the problematization of the public space and formulating wider considerations about similar spheres in other senses and practices.
\end{abstract}

Keywords: neighborhood / city / communication / public space / subjectivity 


\section{Introducción}

$\mathrm{E}$ n el presente artículo proponemos abordar la temática general de la comunicación, la ciudad y el espacio público a partir de la problematización de lo barrial. Consideramos que se trata de la dimensión primordial en la construcción de lo público: las territorialidades barriales son producidas en las interfaces del habitar urbano contemporáneo, con cambios de escala y composición según los medios, y soportes diferentes con sus tecnologías y lenguajes específicos. La producción de la llamada cultura barrial es emblemática de la subjetividad y la comunicación de las sociedades urbanas, desde diversos estilos, narrativas y formas expresivas que constituyen un "entorno tema", "un medio mensaje", por demás central en su creación y recreación, y que está presente en la cinematografía, los formatos televisivos (desde informativos hasta novelas), la música popular, el street art; además, en ella se fusiona lo artístico y lo político desde movimientos sociales de diversa índole. Por todo ello es que las esencializaciones en torno a lo barrial son de las más potentes, incluso en tanto dinámica antropológica de naturalización de la existencia. Nuestro interés es, en tal sentido, compartir una serie de consideraciones a partir de la puesta en crisis de esta condición implícita de la espaciotemporalidad de los urbícolas del siglo xxI, con miras a desentrañar el proceso de territorialización que, efectivamente, da forma y sentido a todo tipo de prácticas.
Nos hemos referido hasta aquí a interfases, medios y soportes, tecnologías y lenguajes, estilos y narrativas. Por comunicación se entiende la dimensión de entornos y flujos que transversalmente constituyen la cultura, la sociedad y las relaciones de poder. Llegamos a la problemática comunicacional a partir de la perspectiva del análisis de los procesos de subjetivación, en tanto prácticas y formas de hacer, entre ellas la de hacerse sujeto (Guattari, 1996). La misma definición de subjetividad como mediación, en tanto sinónimo de proceso o devenir, habilita pensar la comunicación como dimensión presente de forma implícita entre las diversas teorías históricas de las ciencias humanas y sociales, central en las concepciones filosóficas más definitorias de nuestro pensamiento.

Esta dimensión comunicacional puede plantearse en dos sentidos complementarios: como la de generación de entornos y la de generación de flujos; ambos se conectan a otros de variada índole, según lo que se conoce como mediación (Martín-Barbero, 1987). De esta forma, todo aquello que "media" entre las cosas, los seres y las entidades de variada índole no constituye un espacio de representación estandarizado por sí mismo, fiel a la transparencia según una suerte de naturaleza dada o, a lo sumo, una línea de desarrollo tecnológico dirigido inexorablemente a garantizar la inmediatez, a pesar de los proyectos biopolíticos que insisten en ello y actúan en consecuencia con el actual "vértigo de las mediaciones" (García Canclini, 2017). Lo comunicacional pone en evidencia 
la importancia de pensar siempre los contenidos en la relación inextricable con formas expresivas, nos exige problematizar los entornos y las mediaciones según lenguajes y narrativas, lógicas de composición que varían en función de todo ello según una comunicación dialógica, heterogénea y polifónica (Bajtín, como se citó en Guattari, 1996). Desde esta perspectiva, que considera la comunicación como dimensión más radical de producción de subjetividad, el sujeto implicado en estos procesos no es un dato previo ni ajeno, sino que se construye en dicho acto comunicativo por la implicación y el atravesamiento simultáneo del que es partícipe. Este proceso nos otorga la consistencia relativa de nuestra existencia, las relaciones entre la territorialización y la desterritorialización (Deleuze y Guattari, 1997). La comunicación urbana, por tanto, se define por las formas de habitar las tramas de la ciudad (Reguillo, 2007), determinadas por géneros y estilos presentes en entornos y mediaciones múltiples. Esto incluye la comunicación intersubjetiva y del paisaje en las calles de la ciudad, cada vez más digitalizada en su diseño y cotidianidad (De Kerckhove, 2001), a las representaciones y los imaginarios que las sustentan, sea en la literatura, la cinematografía, la televisión, y según las diferentes redes sociales y sus interconexiones (García Vargas y Román Velázquez, 2011).

En consonancia con esta línea de análisis, en la articulación de la antropología, la arquitectura y la comunicación (Álvarez Pedrosian, 2011), consideramos pertinente descomponer el llamado espacio público para comprender muchos de los procesos experimentados en las ciudades contemporáneas. Si tomamos en cuenta los entornos o los ambientes, los flujos o las mediaciones, nos encontramos con "espacios-y-públicos" específicos, o sea, con la conjunción de dos tipos de entidades de naturaleza diferente, ensambladas a su vez en formas singulares según las características de cada configuración socioterritorial (Álvarez Pedrosian, 2014). Ello es aún más evidente cuando de espacios virtuales se trata, o diversos públicos generados en semiosferas y procesos de mediación ligados a ellos. Las narrativas multimediáticas se instalan entre espacios heterogéneos, ligados por agentes, fuerzas y procesos que tejen tramas comunicacionales (Reguillo, 2007; Álvarez Pedrosian, 2016) y confeccionan los universos de existencia de los que somos parte como habitantes, ciudadanos, público.

Como fenómeno de gran presencia en América Latina, ligado históricamente a otros espacios culturales como el Mediterráneo y, más antiguamente, el Oriente Próximo, las diversas formas de lo barrial han caracterizado algo así como la dimensión más concreta para los habitantes. Estos territorios y sus territorialidades han surgido en la inmanencia de la vida cotidiana en algunos casos y momentos, han sido proyectados desde la planificación urbana para retomar preexistencias o como propuestas nuevas; incluso a veces son negadas o desconocidas, o se trabaja para su erradicación, principalmente, 
desde ciertos agentes públicos $\mathrm{y}$ privados que diseñan la ciudad.

Lo que se considera como barrio, su existencia como territorio donde se materializa lo que se concibe como lo público más próximo en determinada sociedad, ha sido objeto de estudios etnográficos de gran influencia a lo largo de la historiografía moderna y contemporánea (Gravano, 2003). Nuestro trabajo pretende reflexionar sobre las cualidades y dimensiones de lo barrial como mediación compleja en la cual el sujeto se define en las tramas de lo que expresa y por lo que queda expuesto, por la cercanía y apropiación en un medio donde lo intempestivo de lo abierto y generalizado irrumpe y produce efectos más o menos deseados, promesas de novedades y revitalizaciones, así como de peligros y riesgos; allí donde lo público es por demás propio y esto propio escapa del control inmediato, el resguardo o la discreción de la intimidad. Más que tratarse de una oposición binaria, lo público y lo íntimo (más que privado) configuran composiciones singulares que merecen ser estudiadas etnográficamente; ámbitos de pliegues y filtraciones, del arte de los tamices y los contrastes, de las heterotopías al mismo tiempo más ordinarias, donde puede aprehenderse el magma de lo social en emergencia permanente (Álvarez Pedrosian, 2018).

Este trabajo se sostiene en diversos estudios etnográficos llevados adelante en contextos disímiles del Montevideo del siglo xxI. Pretendemos extraer consideraciones generales sobre las configuraciones barriales desde allí, para problematizar lo público en la ciudad de cara a las prospectivas volcadas al devenir de un futuro cercano. En vez de pretender reificar el barrio como condición imprescindible para la vida en la ciudad y el derecho sobre ella, la discusión de su naturaleza como territorio construido y en proceso permanente nos permite valorar aún más los atributos que tradicionalmente se le asignan, al mismo tiempo que son considerados críticamente. Repensar la ciudad exige, en definitiva, preguntarse por aquellas territorialidades tradicionales muy activas en la actualidad, con un futuro incierto, plagadas de intensos flujos de información y comunicación que conectan cada vez más a una red multiterritorial diferencialmente estructurada (De Kerckhove, 2001; Haesbaert, 2011; Marcus, 2001).

\section{Expresiones que producen encuentros: comunicación barrial}

Uno de los hechos más sobresalientes en la investigación etnográfica de la periferia montevideana contemporánea, focalizada en la zona de Casavalle (Álvarez Pedrosian, 2013), fue la existencia de conexiones más allá de la fragmentación dominante. Como es sabido, uno de los rasgos centrales de la ciudad capitalista refiere a la lógica de fragmentación que impera en todo sentido, donde los flujos, a su vez, son su contracara (Castells, 1998). Pero estos flujos permitidos no son de cualquier tipo, se trata de aquellos propios 
al sistema del puro intercambio, de la mercancía. Entre complejos habitacionales de bajo costo alzados durante el último medio siglo, áreas de los considerados "barrios tradicionales" de pequeñas chacras y quintas, y los asentamientos irregulares que proliferaron ocupando todo intersticio, tanto entre fragmentos como dentro de ellos (Cecilio, Couriel y Spallanzani, 2003), los habitantes transitan más o menos, incluso cambiando de residencia, aunque no de cualquier forma. Una cierta estabilidad dinámica refuerza los fragmentos replegados sobre sí mismos. En tal situación, la práctica del toque de tambores de candombe es una excepción o, si se quiere, la muestra en acto de cómo existen puentes más allá de estas fronteras contestadas y, en muchos casos, envueltas en una violencia cotidiana muy difícil de soportar.

Con el toque del candombe accedemos a una dimensión donde arte y comunicación no se diferencian. Anotemos brevemente que la misma idea de comunicación no es algo neutro y definido de antemano, sino que es parte consustancial a nuestras exploraciones etnográficas. Este fenómeno nos enseña mucho al respecto y sus rasgos son comunes a otras expresiones culturales provenientes del llamado "Atlántico negro" (Ferreira, 2007) - que baña casi toda América-, fruto de la esclavitud por la que pasaron las poblaciones africanas en manos de los poderes coloniales occidentales. Montevideo albergó un contingente de población de procedencia africana, instalada principalmente en el arrabal de la ciudad, hacia un borde entre el mar y las afueras de la muralla existente por entonces. El conocido Barrio Sur, junto a Palermo (donde la población italiana era mayoritaria, como lo indica su nombre), fue el territorio de configuración de la cultura afrouruguaya por excelencia. Pero el candombe, como expresión artística y cultural más amplia, estuvo presente incluso en otras ciudades y localidades de Uruguay (otrora Banda Oriental, por su ubicación en relación con el río correspondiente), con una gran importancia en la ciudad puerto. Buenos Aires tuvo su propio candombe, lo que refiere a un fenómeno regional rioplatense más allá de las fronteras nacionales posteriormente fijadas. Lo cierto es que, en las décadas finales del siglo pasado, fue un elemento significativo de la apertura democrática tras la última dictadura cívico-militar (1973-1984), en tanto dinámica contracultural que sufriera una dura represión materializada en la demolición de los tradicionales conventillos (Trigo, 1997). Su expansión como manifestación territorial barrial, ligada también con posterioridad a las dinámicas de jóvenes y adolescentes en la ciudad - al igual que en otras latitudes- (Garcés Montoya, 2005), las políticas culturales asociadas al carnaval y sus festejos, así como otros factores, ha generado una proliferación de comparsas o, a lo sumo, bateas por toda la ciudad capital y en diversas zonas urbanas a lo largo del país.

Unos más improvisados, otros con grados de organización considerables, los colectivos de tambores salen semanalmente a llenar de sonidos y bailes las calles. En procesión, siguiendo un 
conjunto de instrumentos de percusión generadores de una polirritmia específica, esperados y seguidos por vecinos y allegados que bailan a su compás, las agrupaciones desfilan por el espacio urbano llenando de sonoridad el aire. Resulta imposible controlar los efectos de ello en nuestros cuerpos, la invitación a bailar con más desenfreno o la atención de un músico profesional fascinado por lo que está ejecutándose allí mismo. Las energías colectivas son liberadas y componen una movilización vital de neto corte barrial (Gonçalvez Boggio, 2007), la cual anima cualquier entorno pauperizado o vaciado por la homogeneización del tener y el no tener, por los efectos de la presencia aplastante del capital o su ausencia. Demás está decir que, como toda expresión cultural popular, el candombe se debate con las políticas culturales y la mercantilización mediática de sus rasgos más genuinos, ante el peligro del vaciamiento de contenido, de la conversión de sus valores de uso en valores de puro cambio, en los términos del pensamiento crítico. Pero, así y todo, en las calles, sendas y pasajes de los barrios más desfavorecidos y en los de los sectores medios de la sociedad, los tambores de fin de semana son un espectáculo montado por los propios vecinos y gestionado de tal forma. Cuando nos encontramos con territorios urbanos donde la fragmentación es radical y los límites son contestados, se experimenta esa sensación de invasión ante el otro alguna vez recién llegado (Elias y Scotson, 2016) (esto puede haber sucedido décadas o incluso siglos atrás, pero sus efectos han perdurado). La existencia de estas formas de expresión, o sea, de comunicación y arte, poseen un valor incalculable. Los tambores de candombe son, según nuestra etnografía, de las pocas cosas que logran atravesar y ligar estos mundos, replegados sobre sí mismos, en un típico paisaje en forma de patchwork propio de las periferias urbanas contemporáneas (Álvarez Pedrosian, 2016).

¿Existen otros fenómenos similares en su accionar y potencialidades? Muy pocos, lamentablemente. Se pueden encontrar otro tipo de actividades que concentran, articulan y ponen en conexión a las subjetividades involucradas, pero el énfasis en los intereses particulares y las necesidades concretas son dominantes. Este es un rasgo general de cualquier actividad humana; es importante no pensar que es posible separarlos, aislar algo así en acciones desinteresadas. Estas expresiones o formas de comunicación están atravesadas por relaciones de fuerza, como ocurre en general, que siguen la tríada saber/ poder/subjetividad (Deleuze, 1987). De lo que se trata, más bien, es de poder identificar fenómenos que, a pesar y gracias a ello, logran establecer vínculos, generar posibilidades para nuevos encuentros, transversalizar $y$, con ello, desterritorializar para volver a crear nuevos territorios (Guattari, 2008). Estas prácticas, por tanto, serían las más importantes para la producción de subjetividad urbana tendientes a la integración social. No se trata de homogeneizar, sino de coser lo que las 
dinámicas imperantes se obstinan en separar y enfrentar una y otra vez.

Otro ejemplo de nuestros trabajos etnográficos puede servirnos de igual forma para aportar a una conceptualización de lo barrial como dimensión privilegiada a la hora de pretender comprender las dinámicas urbanas, la producción de subjetividad y los desafíos antes los que nos enfrentamos actualmente. En el marco de la investigación sobre las formas de habitar, las narrativas y prácticas espaciales existentes en una de las zonas más consolidadas de la ciudad de Montevideo, su segundo ensanche histórico - conocido técnicamente en el último tercio del siglo XIX como Ciudad Novísima (Altezor y Baracchini, 1971; Castellanos, 1971; Carmona y Gómez, 2002)-, nos hemos encontrado con diversos procesos al respecto. Uno de ellos está asociado a prácticas comunicacionales según dinámicas de producción de subjetividad donde las otredades, las distancias y las cercanías, las identificaciones múltiples desbordan clasificaciones sociales relativas a lo que es considerado como normal y anormal. Se trata de los vínculos existentes entre quienes comparten el entorno inmediato con el entorno psiquiátrico más antiguo, conocido como el hospital Vilardebó.

Gracias a un dispositivo complejo de diversos equipos de estudiantes universitarios, así como de habitantes convocados en espacios alternativos de escritura e investigación y otros colectivos sociales autogestionados, hemos podido indagar al respecto. Se trata del hospital psiquiátrico más antiguo de la ciudad y el país.
Ubicado en un predio otrora parte de la quinta familiar del médico por el que lleva su nombre, su tipología arquitectónica es la típica de aquellas "instituciones totales" (Goffman, 1984) del siglo xIx. No se trata de un panóptico, tal como las analizara Foucault, para dar cuenta de las relaciones entre saberes y poderes en la producción de subjetividad, pero su funcionamiento histórico fue del mismo estilo, normalizador y negador de las singularidades de quienes allí terminaban encerrados (Foucault, 2000).

Por cuestiones topográficas y de propiedad, fue conformándose una de las pocas macromanzanas que existen en el seno de la ciudad consolidada, con el curso cercano del arroyo Seco - entubado bajo la superficie- y sus diversos ramales, desde las primeras décadas del siglo xx. En los años de la última dictadura cívico-militar que asoló a Uruguay, se tomó el amplio fondo, casi salvaje, de vegetación y se erigió un complejo habitacional para sectores medios. El llamado complejo Zapicán sigue una tipología cálida, de bloques de mediana altura, de ladrillos a la vista, según lo mejor del modelo de la arquitectura internacional ajustado a las críticas de los sucesores del movimiento en procura de otra calidad ambiental, según una espacialidad que a un tiempo persigue los valores de la higiene, el bienestar y el confort, pero que reconoce otro tipo de necesidades, como las de dotar de singularidad a la materialidad. Una serie de calles organiza el complejo edilicio en tupidos jardines entre los bloques, con elementos de alto valor paisajístico. 
Los apartamentos no fueron solo habitados por simpatizantes del régimen, sino por familias de sectores medios tradicionalmente consolidadas, ligadas al ejercicio de profesionales liberales, quienes encontraron allí una solución para la crisis general que atravesaba la sociedad uruguaya y que empujaba a la población hacia la periferia o más allá, conforme con un proceso de disgregación territorial de mayor alcance temporal. Para los sectores altos, por supuesto, no había mayores inconvenientes, y la migración hacia el este sobre la faja costera, emprendida ni bien la ciudad se fue democratizando, siguió su curso. Los sectores más desfavorecidos de la sociedad fueron quienes padecieron los mayores estragos; fueron ellos los que, a la postre, nutrieron dicha periferia pauperizada a la que hacíamos referencia más arriba. Mientras tanto, estos sectores medios se debatían en una ciudad en decadencia, una sociedad gobernada por un régimen neofascista y una planificación urbana inexistente (Couriel, 2010).

Es así que el complejo se erigió compartiendo dicha gran manzana con el hospital psiquiátrico, y en momentos nada sencillos. Gracias al análisis del paisaje urbano, las prácticas espaciales actuales y los relatos e historia de vida de sus pobladores, nos encontramos con que las relaciones entre residentes y pacientes internados, en particular, fueron intensas y productivas. Diversos tipos de fronteras se materializaron a lo largo de los casi cuarenta años que lleva el complejo habitacional en pie. Vallas cortas, una zona de huertas que se mantuvo de parte del hospital, canchas de fútbol y otros deportes del lado del complejo habitacional y de otro de los espacios colindantes (revalorizado recientemente con el nombre de Rincón del Reducto) refieren a fuertes vínculos entre los considerados socialmente como locos y los habitantes rescatados, en su momento, de terminar en situaciones de mayor precariedad por la migración cuasi forzada hacia los márgenes de la ciudad y más allá. Los internos a los que se les permitía salir en ciertos momentos del día generaban lazos de amistad sostenidos en relaciones de vecinazgo bastante particulares. Especialmente para pedir yerba mate, tabaco de liar, algún alimento $\mathrm{u}$ otro tipo de bien, como alguna prenda de vestir, se tejían relaciones de intercambio, aunque francamente unidireccionales. Podemos referirnos a estos vínculos como formas del "don", del dar-y-recibir entre los involucrados, $y$, en tal sentido, a una de las concepciones de comunicación más clásica proveniente de la etnología (Mauss, 1979), en la que las relaciones no se reducen a la simple mercantilización, ni siquiera a la caridad en un sentido burgués. La dinámica del don es considerada una de las fuentes principales en la concepción de lo comunicacional. Ante los temores y prejuicios generados por doquier al respecto, quienes habitaban el complejo hacían alarde de una tolerancia y amplitud de perspectiva más que loable. Luego, con el paso del tiempo, la tendencia general a la fragmentación y el repliegue de 
cada elemento sobre sí mismo también los afectó. De todas formas, existe un debate abierto, nada fácil de zanjar, sobre la necesidad de cerrar el complejo a la vía pública, pues, incluso en términos legales, sus calles interiores son consideradas formalmente como espacio público. Esta controversia sobre la arquitectura y la ciudad, en tanto materialidades en movimiento (Latour y Yaneva, 2008), envuelve actualmente a la comisión organizada de los habitantes. Pero no refiere para nada a la situación de vecindad con el psiquiátrico, sino que atañe al clima general de inseguridad que orienta los imaginarios urbanos contemporáneos y las asociaciones que se realizan entre pobreza, violencia y espacios públicos (Low, 2003; Kruijt, 2008).

Concomitante con ello, el hospital experimenta una serie de fenómenos que lo van transformando; en especial, la práctica de un colectivo de profesionales e internos que llevan adelante la audición Radio Vilardevoz (por la conjunción de las palabras Vilardebó y voz). Se trata de un ejemplo de las experiencias terapéuticas radiofónicas en hospitales de salud mental, tan importantes para avanzar hacia un modelo de desmanicomialización (véase su web: http://www. vilardevoz.org/). Es así que se comenzó a emitir del hospital hacia el entorno cercano, barrial; más recientemente, se hace también a través de internet. El colectivo involucrado ha alcanzado grandes logros y se ha articulado con experiencias internacionales de similar magnitud.
A nivel nacional, el debate sobre una nueva ley de salud mental está sobre la mesa, y la transformación añorada de este tipo de espacios de encierro en otra propuesta sigue en marcha a pesar de avances y retrocesos. Incluso no es fortuito el hecho de que, desde el pensamiento proyectual, exista la idea de reconvertir esta antigua macromanzana en un complejo de espacios verdes para el uso de la población, aunque sea una de las propuestas que menos cabida ha tenido, de un conjunto de planteos esbozados, en los momentos de la reapertura democrática, en el marco de un emblemático laboratorio de ideas urbanas (Sprechmann et al., 1986). En estos momentos, la entrada principal del hospital muestra un rostro difícil de asimilar: varios indigentes duermen con sus colchones allí y en las proximidades, frente a las rejas de las que cuelgan pancartas sindicales de los trabajadores del campo sanitario en permanente conflicto con las autoridades, tras lo cual se yergue la aguja y se extienden las galerías decimonónicas del nosocomio, con amplios sectores de la construcción en ruinas.

Aquella máxima de la comunicología que reza "no se puede no comunicar" (Watzlawick, como se citó en Winkin, 1982) es central respecto de nuestras temáticas, pues se articula con otros enfoques en el interés común en la comunicación como dinámica mediacional de producción de subjetividad. Es imposible habitar la ciudad sin comunicarse, sin ser afectado y afectar al entorno del cual se es parte, y sin emitir mensajes 
hacia otros entornos posibles y virtuales. Ciertamente, están el desarrollo tecnológico y el interés por sostener estructuras donde parece posible aislarse completamente del otro. Es el caso de las zonas cerradas de una ciudad, con vigilancia digital omnipresente, controles por doquier y demás (Low, 2003). Pero, así y todo, resulta siempre insostenible. Esas burbujas espaciotemporales de exclusividad son presionadas y estallan, sea como sea, desde el exterior coextensivo o desde sus entrañas, ya que no se pueden sostener sin la presencia de recursos de todo tipo, sin trabajadores oriundos del afuera, por ejemplo. Es cierto que seguimos obsesionados por ese camino, y el hecho de que América Latina sea la región del planeta más desigual de todas puede apreciarse plasmada en esta dinámica socioterritorial, a una vez causa y efecto del proceso de segregación residencial. Pero la interpelación de los otros se cuela por todas las aberturas, sean microscópicas o inmensas, sean cercanas o corridas más y más lejos.

La lógica de una ciudad que no atiende las texturas que la conforman atenta contra sí misma, siempre es solo cuestión de tiempo. El gasto en vidas y energías puestas en el desarrollo y sostén de tecnologías de variada índole (desde la arquitectura hasta las ondas de radio y los sistemas de video integrados) no pueden contener el torrente comunicativo de procesos inter- y transubjetivos inevitables. Pretender negarlo es lo que nos conduce a una espiral mortífera de mayor fragmentación, desigualdad y represión hasta montar una realidad a punto de estallar en pedazos. Frente a esto, y sin caer en simplificaciones idealizantes, aquellos espacios urbanos cargados de calidad por las tramas de seres, objetos, entidades de variada índole, expresadas en medios y soportes heterogéneos entrelazados, nos habilitan la experiencia estética central en el habitar (Heidegger, 1994; Álvarez Pedrosian y Blanco Latierro, 2013). Cuando ello es posible, siempre en conflicto, según tensiones productivas de subjetividad, es cuando sentimos que estamos viviendo en una ciudad dinámica, habilitadora de posibilidades para sus habitantes, que nos comunica desde el paisaje que nos envuelve con su atmósfera y en el que se encarna (Jóhannesdóttir, 2010) que es posible crear y crearse en la condición dialógica de los encuentros.

\section{Entre lo concreto y lo abstracto: las escalas desde la ciudad barrial}

Repensar la comunicación, la ciudad y el espacio público desde las territorialidades barriales nos permite divisar algunas dinámicas que de otra manera resultan difíciles de aprehender o que incluso se encuentran invisibilizadas, pero que hacen un nudo gordiano de esta encrucijada. No pensemos al barrio como un área homogénea, circunscrita, unidimensional. Cuando no logramos extrañarnos lo suficiente, volvemos a reafirmar esencialismos al estilo de territorios exclusivos y excluyentes, reproduciendo la fragmentación de un tipo de relativismo antropológico de 
escasa proyección filosófica. Cuando estamos en los barrios estamos en las ciudades, y no en una sola, sino en una red que nos conecta a una heterogeneidad incluso continental y planetaria; se trata de la multiplicidad territorial (Haesbaert, 2011), con fuerza suficiente como para dislocar distinciones binarias al estilo de lo local y global (Massey, como se citó en Tapia, 2013).

En el marco de las investigaciones etnográficas a las que hemos hecho referencia antes, desplegamos diversos dispositivos de participación con sus respectivos alcances cognoscentes. Entre ellos, llevamos a cabo un taller de narrativa e investigación sobre la ciudad en un clásico club barrial, abierto a todo aquel habitante que quisiera inscribirse. En tal sentido, consideramos fundamental ejercitar una etnografía colaborativa, con la que poder producir conocimiento con y entre los sujetos que directamente son parte de los fenómenos en cuestión (Lassiter, 2005). Esto no inhabilita las diferencias que pueden existir dadas las formaciones, las habilidades y los saberes preexistentes, más bien todo lo contrario. Lo que se busca con este tipo de estrategias teórico-metodológicas es disponer de un ámbito de diálogo inter- y transcultural en el seno mismo de la investigación sobre tales asuntos. Es así que, entre otros entornos articulados de exploración en temáticas del habitar urbano contemporáneo, pudimos sostener este espacio colectivo de aprendizaje con habitantes de perfiles muy variados, todos comprometidos con el ejercicio de la ciudadanía $\mathrm{y}$ activos en relación con las prácticas culturales que instituciones de tipo municipal les proponen, en alianza con diversos agentes locales (en este caso, una biblioteca y una editorial artesanal funcionando por entonces en un club social y deportivo histórico de la ciudad de Montevideo).

Este no es el sitio para profundizar en este tipo de experiencias por demás significativas, pero siguiendo el hilo argumental de este artículo, lo crucial aquí es retomar el carácter de la mirada que pudimos producir entre todos en tales circunstancias. Los ocho casos sobre los que realizamos el trabajo de campo etnográfico, el análisis de fuentes secundarias y el acercamiento a temas antropológicos y urbanísticos fueron pensados y definidos en conjunto, en el marco de nuestra propuesta sobre el estudio de los procesos de subjetivación, las narrativas y las prácticas espaciales referidas a los territorios del segundo ensanche de la ciudad. La aparición de nuevos modelos para el diseño de plazas y parques - considerados como integradores en los últimos años-, los dilemas en torno a las infraestructuras ferroviarias para pasajeros y la cuestión de la movilidad y la segregación residencial, la presencia del trabajo en la ciudad y sus relaciones con otras prácticas cotidianas, las experiencias estéticas propiciadas por aquellos espacios en principio pensados para el ocio y el esparcimiento, las relaciones conflictivas establecidas entre urbícolas y la naturaleza negada desde la perspectiva higienista del siglo pasado pero insistente en su presencia, las transformaciones de zonas verdes 
por el tránsito aplastante a escala urbana y regional, la materialización de proyectos en edificios de programas especiales -como el primer jardín de infantes de toda América Latina- y, por último, la historia de vida entramada en la historia de un edificio en una esquina emblemática de la ciudad fueron las temáticas abordadas. Ello, a su vez, fue realizado en articulación con los otros espacios de investigación y enseñanza que coordinamos. Cuando llega el momento de definir, también colectivamente, el nombre de la publicación artesanal (que integrará el libro de estilo académico más amplio, en proceso actualmente), los participantes llegamos al título Narrativas barriales. La pregunta que inmediatamente se nos presentó y que volcamos en el taller con los participantes fue la siguiente: ¿qué es lo que se pretende expresar, comunicar con ello?

A partir de la etnografía colaborativa no se disuelven las distinciones entre perspectivas, sino que se trata de componer un conocimiento colectivo rico en la heterogeneidad de puntos de vista. En particular, esto es crucial para salir de los estancamientos a los que pueden conducirnos los ejercicios puramente academicistas, en los que la teoría social y su filosofía resultan completamente ajenas al universo de fenómenos y problemáticas que se pretenden abordar. La antropología tiene una historia por demás rica en tal sentido (Lassiter, 2005), lo mismo sucede con la comunicación en sus vertientes - denominadas a vecesalternativa, comunitaria, popular, $\mathrm{u}$ en otras corrientes que se han ido planteando principalmente desde el trabajo con los sectores más desfavorecidos socialmente y que, a pesar de todo, no encuentran un reconocimiento académico del todo satisfactorio (Barranquero y Sáez Baeza, 2010). Romper con los estilos de pensamiento que tienden a la pura abstracción para dar paso a una dialógica crítica en medio de las tensiones de lo social implica un gran desafío epistemológico, político y comunicacional. No alcanza con decretar la apertura de la mirada, hay que poder sostener el proceso y llegar a resultados tangibles - entiéndase propuestas cognoscentes cargadas de sentidos que se proyecten más allá de lo inmediato, con la mayor amplitud de alcance posible-. De lo contrario, caeríamos en la anécdota de contar con bellos relatos personales de escaso valor para otros que no sean los directamente involucrados, situación que puede ocurrir, en realidad, con cualquier ejercicio de tipo etnográfico (Velasco y Díaz de Rada, 1997). Es así que cada trabajo emprendido fue tensionado para producir este efecto proyectivo - de trascendencia en cuanto generación de conocimientodesde lo situado y contextual de las experiencias propias de cada participante, según sus formaciones, deseos y expectativas, que van del trabajo en un organismo municipal a una cooperativa de transporte urbano, pasando por la medicina, la declamación o la poesía, en diferentes grados de profesionalización, según géneros, edades y características socioeconómicas de un amplio espectro.

Estas narrativas barriales son producidas por y productoras de lo que hemos 
denominado "saberes habitantes", constructos culturales que habitan la ciudad como entidades transversales que se encarnan en subjetividades, se materializan en todo tipo de objetos y movimientos; son algo así como lo urbano mismo existiendo en prácticas cotidianas. Nosotros trabajamos procurando tomar las nociones y los prejuicios con los que llegamos como parte de los imaginarios sociales urbanos presentes y, desde allí, abrirlos a la posible creación conceptual, gracias a la exploración etnográfica en territorios tan propios como ajenos, comenzando con el ejercicio de la cartografía social (Diez Tetamanti y Escudero, 2012). El resultado de esta dinámica productiva generó narrativas que, a pesar de las crisis y problematizaciones en cadena sobre dichos presupuestos de partida, fueron calificadas como de barriales por los participantes. En la tensión entre el distanciamiento y la inmersión en los fenómenos de estudio, la etnografía trabaja desnaturalizando lo que socialmente se establece como lo dado, para con ello brindar nuevas visiones relativas a dichas realidades (Lins Ribeiro, 1998). Pero para mantenerse fieles a sus singularidades, nuestros participantes y colaboradores del taller de narrativas urbanas optaron por poner énfasis en el carácter barrial de estas producciones. Lo que allí está en juego es otra mirada sobre la ciudad, nacida desde lo emergente de sus habitantes, invitados a experimentar con la producción de conocimiento e hibridarse con teorías, métodos y técnicas de un campo científico determinado; sin embargo, no por ello niegan lo que consideran más genuino en el mismo gesto combinatorio. De esta manera, lo barrial aparece como una experiencia, una perspectiva, un agenciamiento (Deleuze y Guattari, 1997) desde el cual se proyecta una visión más amplia, pero sin desconocerla.

Lo que consideramos relevante para los fines de este artículo es la coexistencia de diversas escalas, cada una con sus lógicas, y en particular dos direcciones y sus tipos ideales: por un lado, la abstracción de la mirada cenital de la ciudad como un todo, asociada a los técnicos de la planificación y las políticas urbanas, $\mathrm{y}$, por otro, la de lo concreto del habitar cotidiano, expresada en la vida de los habitantes. Podemos pensarlas como opuestas, pero no es el mejor de los escenarios si queremos generar experiencias de participación ciudadana que además den frutos comunicacionales y cognoscentes que aporten herramientas para redefinir las problemáticas y encausar nuevos desafíos. En un clásico de los estudios culturales urbanos, De Certeau (2000) elaboraba su famoso relato desde la visión que ofrecía el piso 110 del Word Trade Center de Nueva York, haciendo alusión a estos dos modos de aprehender nuestros entornos de vida en tanto urbícolas. Si no fuera por esas alturas y las perspectivas que habilita, el habitante medio no tendría oportunidades para experimentar esa suerte de totalización, desde lo altísimo, donde no se percibe a sus conciudadanos, pero se los puede intuir por el movimiento de objetos, como los vehículos por las vías de tránsito, y tras las minúsculas ventanas de 
los grandes edificios. Consideramos que, en las últimas décadas del siglo pasado y comienzos de este milenio, distinciones tan tajantes como estas se han visto alteradas gracias a trabajos y planteos como los realizados por estos grandes pensadores.

¿No son acaso los propios planificadores y diseñadores los decisores de políticas públicas para la ciudad, y otros agentes, habitantes como todos los demás, con una vida por dentro de esa trama que se divisa allí abajo, a lo lejos? Lo abstracto y lo concreto se relacionan de maneras no dicotómicas, o por lo menos está el potencial para que esto no ocurra si bien la tendencia del racionalismo de tradición occidental ha hecho hincapié en este tipo de estructuras. Por supuesto, no se trata de negar la capacidad de objetivación generada por las tomas de distancia, sino de comprender cómo siempre existen conexiones necesarias con lo inmanente; en este caso, con lo más profundo de lo cotidiano de nuestra vida en la ciudad. Es así que debe pensarse en lo abstracto y lo concreto como tendencias que además son dinámicas, incluso como postas en un trayecto intelectual y afectivo donde vamos jalonando instancias de un proceso (Álvarez Pedrosian, 2013), sea investigativo, asociado a la producción de conocimiento como fin primero, o como parte de nuestros saberes cotidianos para la resolución de problemas, entre tácticas y estrategias - para retomar las nociones de De Certeau (2000) - . Cuando se puede aprender sobre la ciudad desde la mirada barrial de los habitantes, más cuando esta es exigida por ejercicios como el desarrollado en nuestro taller etnográfico, se fomenta y sostiene la generación de abstracciones nuevas potenciando las existentes previamente.

Podemos decir, por tanto, que lo barrial es la ciudad desde su dimensión "molecular" (Deleuze y Guattari, 1997). Ante lo "molar", no hay una simple oposición, sino un cambio de naturaleza a un tiempo coexistente, lo cual la dualidad entre lo "micro" y lo "macro" no parece permitirnos pensar. Los habitantes que participaron del ejercicio de investigación hicieron alusión a esta mirada desde abajo, desde dentro, con proyección más amplia hacia una totalidad, pero la cual es abierta, como cualquier tipo de ciudad, proyectada desde la particularidad de una experiencia urbana directa que es catalogada como barrial. Creemos que con esto se pueden realizar aportes significativos para comprender los fenómenos de la comunicación urbana, insistimos, sin negar los poderes que conllevan perspectivas analíticas desarrolladas durante los últimos siglos, sin caer en una suerte de nuevo sustancialismo en nombre de la voz verdadera de los habitantes. Se trata más bien de dar paso a la complejidad, la multiescalaridad de estos procesos. En tal sentido, más que quedarnos en tal o cual nivel, para nada paralelos o circunscritos unos dentro de otros, se trata de poder procesar una experiencia cada vez más cotidiana: la de habitar, a un mismo tiempo, en múltiples universos transversales con sus propias lógicas territoriales, sus escalas y las dinámicas que nos llevan entre abstracciones y concreciones, más o menos aprehendidas, pero 
más que presentes en nuestros procesos de subjetivación contemporáneos. Basta con pensar en lo que ocurre en las megalópolis planetarias para captar un poco más aquello a lo que nos referimos. La cotidianidad entre inmensas estructuras edilicias, sistemas de transporte que mueven decenas de millones de citadinos por día, conectados a su vez a nubes virtuales de información (De Kerckhove, 2001), nos tienen envueltos en dinámicas en las que lo tradicionalmente pensando como "escala humana" es redefinido sin cesar; incluso, en ciertas circunstancias, lo que se percibe y siente es de muy difícil captación para determinada subjetividad, sea por no habitar cotidianamente entre estos flujos, en principio más desterritorializantes, o por una dinámica permanente en la que los cambios tecnológicos transforman la misma concepción del espacio-tiempo.

Desde este punto de vista, el barrio y la ciudad no se oponen. Es necesario pensar en la ciudad barrial, en esa abstracción desde las concreciones que podemos encontrar en el habitar, apoyados en estrategias etnográficas para su abordaje. Lo barrial, como cualidad incluso, puede hallarse de formas tan heterogéneas e híbridas donde los flujos de desterritorialización son tan intensos que parecen disolver el territorio, como en el caso de aquellas zonas de extrema articulación de fuerzas globales, asociadas directamente al capitalismo y su "sistema mundo" (Wallerstein, 2004; Marcus, 2001). Ciertamente, existen muchas voces que nos hablan de la pérdida de la ciudad vivible, de espacios tomados excluyentemente para la disposición de capitales económicos, infraestructuras de variada índole, ambientes de puro intercambio donde no es posible hacerse un lugar (Tuan, 2003; Augé, 1994). En todo caso, poder enfrentarse a estos retos de nuestras ciudades contemporáneas requiere desplegar una perspectiva que no recaiga en esencializaciones al estilo de los "arraigos" como única condición para definir nuestras espacialidades (Choay, 2009), a un tiempo que no se permita el juego fortuito de intereses desprovistos del "derecho a la ciudad" de sus habitantes y los movimientos emancipatorios (Harvey, 2013). Es por todo ello que hemos propuesto que

el barrio puede ser pensado [...] como la dimensión de los flujos de territorialización-desterritorialización, como expresión de la vida urbana, el paisaje de su habitar por excelencia. En él se encuentran, se traducen e intercambian lo abstracto y lo concreto, la forma y el contenido, el adentro y el afuera. (Álvarez Pedrosian, 2018, p. 8)

\section{Conclusiones: el espacio público y lo barrial}

Las territorialidades barriales, más allá de los territorios específicos que podemos identificar como tal o cual barrio, a veces excluyentes unos con otros, superpuestos, implantados, emergidos en la contingencia de la ocupación poblacional y demás, comparten este rasgo de componer un tipo de subjetivación urbana específica. Se trata de aquella en la que el habitante tiene una existencia 
abierta, a un mismo tiempo, a lo intempestivo del afuera -léase las calles y ámbitos de una supuesta libre circulación-, donde encuentra los elementos para componer la singularidad de su entorno más propio, la intimidad, en sus más variadas formas según las culturas y sociedades de las que se trate (Álvarez Pedrosian, 2018). Esta condición intersticial, para nada lineal $u$ homogénea entre un adentro y un afuera tan solo coextensivo, nos permite problematizar, entre otras cuestiones, la noción misma de espacio público, tan arraigada en los debates en torno al derecho a la ciudad, las políticas urbanas e incluso mucho más allá en lo relativo a la concepción de ciudadanía en cualquier tipo de esfera comunicacional (Martín-Barbero, 2010).

Como se planteó en la introducción de este trabajo, los entornos espaciotemporales considerados como barriales -en sus más diversas acepciones- nos permiten analizar los procesos urbanos desde la propia lógica construccionista de su composición antropológica. Las mediaciones complejas que podemos encontrar desde su abordaje etnográfico, o sea, las formas comunicativas que se entretejen entre las prácticas de habitantes y agentes de variada índole, ejemplificadas brevemente en los casos expuestos, nos muestran cómo no podemos reificar los territorios que habitamos, ya que no son un simple fondo para nuestras vidas, sino la trama misma que les da su consistencia (Ingold, 2013). Cuando se apela al espacio público es fácil caer en la naturalización de normas, conductas y prácticas, que resultan algo así como un deber ser impuesto coercitivamente como pacto obligado de convivencia (Delgado, 1999, 2007). Por el contrario, creemos que, si existe algo así como ese ámbito de intercambios y encuentros, libre pasaje y escenario de manifestaciones de las más diversas subjetividades, es gracias a que se produce desde la inmanencia de los procesos en juego.

El espacio público es producido, es creado contingentemente y no posee, por tanto, una definición a priori o fuera de contexto. Cuando se ha tomado por el camino inverso, se ha llegado a situaciones de control social que rozan o son definitivamente mecanismos de opresión. Algunos análisis llegan incluso a plantear su no existencia o, a lo sumo, su condición de resultado directo de una ideología liberal occidental que se proyecta sobre lo urbano (Delgado, 2007). El espacio público es tan propenso a estas generalizaciones que incluso puede confundirse con la noción misma de lo social, como su sinónimo, lo que lo hace incluso inasible (Álvarez Pedrosian, 2014). Parecería que es una entelequia desprovista de conflicto, regulada en todas direcciones, $\mathrm{o}$, por el contrario, un ámbito de pura espontaneidad donde no hay que regular absolutamente nada para que tenga lugar (Sanmiguel, 2005). Se produce una suerte de ensamblaje entre entidades diferentes, el cual, a su vez, elimina toda singularización y heterogeneidad: se pasa de espacios y públicos, definidos ambos por cualidades que hay que precisar en cada caso, a un espacio público genérico tendiente, una vez más, a la abstracción 
pura (Álvarez Pedrosian, 2014). Es por ello que los fenómenos barriales nos permiten tener otro tipo de acercamiento a esta problemática al encontrarnos con las dinámicas de producción del espacio y sus mediaciones comunicacionales. Gracias a una mirada barrial con pretensiones de proyección urbana, es decir, focalizada en lo concreto de la vida de los habitantes, pero sin reducirse a los constreñimientos de lo local como una suerte de aquí-y-ahora encapsulado en lo "micro", es que podemos aprehender una serie de fenómenos urbanos, desde su dimensión comunicacional, que nos conecta con los procesos constitutivos del habitar (Heidegger, 1994).

No pretendemos afirmar que sin barrios no hay una vida digna de ser llevada a cabo o que fomentar los barrios es la única forma de garantizar el goce de derechos y la amplitud de condiciones para la mayor parte de la población. Pueden existir otras formas de llevar esto adelante, no podemos cerrarnos en tal sentido. Pero lo cierto es que la vida de barrio, tal como se la concibe desde hace siglos en nuestras civilizaciones, nos ha dado la posibilidad de construirnos como habitantes de un mundo rico en sus texturas, donde es posible la conformación de la subjetividad, a un mismo tiempo abierta más allá de las limitaciones inmediatas y soberana en algún tipo de territorio considerado de esa manera como propio, e incluso ir más allá para, en diferentes grados y sentidos, alcanzar a territorializarse en otras dimensiones en principio totalmente desconocidas. Se trata de un arraigo, es cierto, pero no necesariamente del tipo esencialista. Es, más bien, una forma de construir "focos autopoiéticos de producción de subjetividad" (Guattari, 1996) desde el residir como base de toda existencia. Puede haber territorialización al mismo tiempo y gracias a los flujos de desterritorialización que se reconocen en su seno, en tanto líneas de fuga que componen todo territorio (Deleuze y Guattari, 1997). Se trata, por tanto, de una forma de cosmopolitismo cualificado, de un habitar en tanto ser-en-el-mundo que no recae en la fijación sustancialista que, como sabemos, lleva casi siempre a la justificación de la violencia de unos contra otros en nombre de patrias exclusivas y excluyentes.

Tomemos en cuenta, por tanto, la riqueza de composiciones de lo barrial para desnaturalizar el espacio público, sea en la esfera que sea, no solo en la vida urbana. Gracias a ello, podremos ir consolidando caminos para, a un mismo tiempo, aportar en la construcción de una vida mejor para todos sin la necesidad de recurrir a discursos normativos, al deber ser que aplasta al ser. Las artes de los tamices y los contrastes, como hemos dicho, nos enseñan acerca de la forma de tejer la convivencia, de crear territorios siempre singulares y singularizantes, a la vez conectados más allá por flujos que atraviesan una densa red de mediaciones comunicacionales más abstractas o más concretas según la perspectiva y la experiencia suscitada, trama que no es otra que la de la propia vida en sus más variadas formas. 


\section{Referencias}

Altezor, C., y Baracchini, H. (1971). Historia urbanística y edilicia de la ciudad de Montevideo. Desde su fundación colonial hasta nuestros días. Montevideo: Junta Departamental de Montevideo.

Álvarez Pedrosian, E. (2011). Espacialidades: antropología, arquitectura y comunicación. Actas electrónicas de la IX Reunião de Antropologia do Mercosul. Curitiba: Universidade Federal do Paraná. Recuperado dehttps://eva.udelar.edu.uy/pluginfile.php/831785/ mod_folder/content/0/\%20Espacialidades\%20antropolog\%C3\%ADa\%2C\%20 arquitectura\%20y\%20comunicaci\%C3\%B3n\%20-\%20\%C3\%81LVAREZ\%20 PEDROSIAN.pdf?forcedownload=1

Álvarez Pedrosian, E. (2013). Casavalle bajo el sol. Investigación etnográfica sobre territorialidad, identidad y memoria en la periferia urbana de principios de milenio. Montevideo: CSIC-Udelar.

Álvarez Pedrosian, E. (2014). La gestación de un territorio o de cómo se tejela convivencia. En Actas electrónicas del XII Congreso Latinoamericano de Investigadores de la Comunicación. Lima: Pontificia Universidad Católica del Perú. Recuperado de http://congreso.pucp.edu.pe/alaic2014/wp-content/uploads/2013/09/Ponencia\%C3\%81lvarez-Pedrosian-XII-ALAIC-Lima-2014.pdf

Álvarez Pedrosian, E. (2016). Las tramas socio-territoriales en las que habitamos: aportes para pensar la composición urbana en clave comunicacional. Informatio, 21(2), 69-87. Rcuperado de http://informatio.eubca.edu.uy/ojs/index.php/Infor/article/ view/189

Álvarez Pedrosian, E. (2018). Las territorialidades barriales y sus espacios de creación. En Actas electrónicas del XIV Congreso Latinoamericano de Investigadores de la Comunicación. San José: Universidad de Costa Rica [manuscrito aceptado para su publicación].

Álvarez Pedrosian, E., y Blanco Latierro, M. V. (2013). Componer, habitar, subjetivar. Aportes para la etnografía del habitar. Bifurcaciones. Revista de Estudios Culturales Urbanos, (15). Recuperado de http://www.bifurcaciones.cl/2013/12/ componer-habitar-subjetivar/

Álvarez Pedrosian, E., y Robayna, A. (2013). Devenires escalares en la composición territorial. En Taller Scheps, Escalar 2013 (pp. 242-243). Montevideo: Universidad de La República, Facultad de Arquitectura.

Augé, M. (1994). Los no-lugares. Espacios del anonimato. Una antropología de la sobremodernidad. Barcelona: Gedisa.

Barranquero, A., y Sáez Baeza, C. (2010). Comunicación alternativa y comunicación para el cambio social democrático: sujetos y objetos invisibles en la enseñanza de las 
teorías de la comunicación. Actas electrónicas del Congreso Internacional AE-IC, Málaga. Recuperado de http://www.aeic2010malaga.org/upload/ok/453.pdf

Carmona, L., y Gómez, M. J. (2002). Montevideo. Proceso planificador y crecimiento. Montevideo: Universidad de La República, Facultad de Arquitectura.

Castellanos, A. (1971). Historia del desarrollo edilicio y urbanístico de Montevideo (18291914). Montevideo: Junta Departamental de Montevideo.

Castells, M. (1998). La era de la información (t. I). Madrid: Alianza.

Cecilio, M., Couriel, J., y Spallanzani, M. (2003). La gestión urbana en la generación de los tejidos residenciales de la periferia de Montevideo. Áreas ocupadas por los sectores de población de bajos y medios ingresos. Montevideo: Facultad de Arquitectura-Udelar.

Choay, F. (2009). El reino de lo urbano y la muerte de la ciudad. Andamios, 6(12), 157-187.

Couriel, J. (2010). De cercanías y lejanías. Fragmentación sociourbana del Gran Montevideo. Montevideo: Trilce.

De Certeau, M. (2000). La invención de lo cotidiano 1. Artes de hacer. México: Universidad Iberoamericana.

Deleuze, G. (1987). Foucault. México: Paidós.

Deleuze, G., y Guattari, F. (1997). Mil mesetas. Capitalismo y esquizofrenia II. Valencia: Pre-textos.

Delgado, M. (2007). La ciudad mentirosa. Fraude y miseria del "Modelo Barcelona". Madrid: Los Libros de la Catarata.

Delgado, M. (1999). El animal público. Hacia una antropología de los espacios urbanos. Barcelona: Anagrama.

Diez Tetamanti, J. M., y Escudero, B. (comp.). (2012). Cartografía social: investigaciones e intervención desde las ciencias sociales: métodos y experiencias de aplicación. Comodoro Rivadavia: Universidad de la Patagonia.

Elias, N., y Scotson, J. (2016). Establecidos y marginados. Una investigación sociológica sobre problemas comunitarios. México: Fondo de Cultura Económica.

Ferreira, L. (2007). An afrocentric approach to musical performance in the Black South Atlantic: The Candombe drumming. Trans. Revista Transcultural de Música, (11). Recuperado de https://www.sibetrans.com/trans/articulo/129/an-afrocentricapproach-to-musical-performance-in-the-black-south-atlantic-the-candombedrumming-in-uruguay

Foucault, M. (2000). Los anormales. Buenos Aires: FCE.

García Canclini, N. (2017). El vértigo de las mediaciones. En M. De Moragas, J. L. Terrón y O. Rincón (eds.), De los medios a las mediaciones de Jesús Martín Barbero, 30 años después (pp. 121-123). Bellaterra: InCom-UAB. 
García Vargas, A., y Román Velázquez, P. (2011). Latin American urban cultural studies: unique texts, ordinary cities. Westminster Papers in Communication and Culture, 8(1), 131-153. Recuperado de https://dspace.lboro.ac.uk/dspace-jspui/ bitstream/2134/26683/1/26683.pdf

Garcés Montoya, Á. P. (2005). Nos-otros los jóvenes: polisemias de las culturas y los territorios musicales en Medellín. Editorial Universidad de Medellín.

Goffman, E. (1984). Internados. Ensayos sobre la situación social de los enfermos mentales. Buenos Aires: Amorrortu.

Gonçalvez Boggio, L. (2007). Los territorios del candombe. En Cuerpo y subjetividades contemporáneas. Clínica bioenergética y esquizoanálisis. Montevideo: Clinicabioenergetica.com. Recuperado de http://www.academia.edu/11497032/ Cuerpo_y_subjetividades_contemporáneas

Gravano, A. (2003). Antropología de lo barrial: estudios sobre producción simbólica de la vida urbana. Buenos Aires: Espacio.

Guattari, F. (1996). Caosmosis. Buenos Aires: Manantial.

Guattari, F. (2008). Prácticas ecosóficas y restauración de la ciudad subjetiva. En La ciudad subjetiva y pos-mediática. La polis reinventada (pp. 216-234). Cali: Fundación Comunidad.

Haesbaert, R. (2011). El mito de la desterritorialización: del "fin de los territorios" a la multiterritorialidad. México: Siglo XXI.

Harvey, D. (2013). Ciudades rebeldes. Del derecho de la ciudad a la revolución urbana. Salamanca: Akal.

Heidegger, M. (1994). Construir, habitar, pensar. En Conferencias y artículos (pp. 127-142). Barcelona: Serbal.

Ingold, T. (2013). Making: anthropology, archaeology, art and architecture. Londres: Routledge.

Jóhannesdóttir, G. (2010). Landscape and aesthetic values: not only in the eye of beholder. En K. Benediktsson y K. A. Lund, Conversation with landscape (pp. 109-123). Farnham, Surrey: Ashgate.

Kerckhove de, D. (2001). The architecture of intelligence. Basel: Birkhäuser.

Kruijt, D. (2008). Violencia y pobreza en América Latina: los actores armados. Pensamiento Iberoamericano, (2) [II época], 55-70.

Lassiter, L. E. (2005). The Chicago guide to collaborative ethnography. Chicago: The University of Chicago Press.

Latour, B., y Yaneva, A. (2008). Give me a gun and I will make all buildings move: an ANT's view of architecture. En R. Geiser (Ed.), Exploration in architecture: teaching, design, research (pp. 80-89). Basel: Birkhéiuser. 
Lins Ribeiro, G. (1998). Descotidianizar. Extrañamiento y conciencia práctica, un ensayo sobre la perspectiva antropológica. En M. Boivin, A. Rosato y V. Arribas, Constructores de otredad (pp. 232-237). Buenos Aires: Eudeba.

Low, S. (2003). Behind the gates. Life, security, and the pursuit of happiness in fortress America. Londres: Routledge.

Marcus, G. (2001). Etnografía en/del sistema mundo. El surgimiento de la etnografía multilocal. Alteridades, 11(22), 111-127.

Martín-Barbero, J. (1987). De los medios a las mediaciones. Comunicación, cultura y hegemonía. Barcelona: Gustavo Gili.

Martín-Barbero, J. (2010). Comunicación, espacio público y ciudadanía. Folios [edición especial], 37-51.

Mauss, M. (1979). Ensayo sobre el don. Forma y razón del cambio en las sociedades arcaicas. En Sociología y antropología (pp. 153-263). Madrid: Tecnos.

Reguillo, R. (2007). Ciudad y comunicación. Densidades, ejes y niveles. Diálogos de la comunicación, (47), 1-9.

Sanmiguel, P. E. (2005). Lo privado, lo público, el sujeto y el individuo. Comentarios desde el psicoanálisis. En A. Lozano Ayala (Ed.), Hacia una definición de lo público (pp. 16-24). Bogotá: Universidad Nacional de Colombia.

Sprechmann, T., Bastarrica, J., Otero, R., Kohen, M., Villaamil, A., Gervaz, A., ... y Bervejillo, F. (1986). Propuestas a la ciudad. Montevideo-1986. Montevideo: Taller de Investigaciones Urbanas y Regionales.

Tapia, V. (2013). El concepto de barrio y el problema de su delimitación. Aportes de una aproximación cualitativa y etnográfica. Bifurcaciones. Revista de Estudios Culturales Urbanos, (12). Recuperado de http://www.bifurcaciones.cl/2013/03/elconcepto-de-barrio-y-el-problema-de-su-delimitacion/

Trigo, A. (1997). ¿Cultura uruguaya o culturas linyeras? (Para una cartografía de la neomodernidad posturuguaya). Montevideo: Vintén.

Tuan, Y-F. (2003). Space and place: the perspective of experience. Minneapolis: University of Minnesota Press.

Velasco, H., y Díaz de Rada, Á. (1997). La lógica de la investigación etnográfica. Madrid: Trotta.

Wallerstein, I. (2004). El análisis de los sistema-mundo. En Capitalismo histórico y movimientos antisistémicos (pp. 134-150). Madrid: Akal.

Winkin, Y. (Ed.). (1982). La nueva comunicación. Barcelona: Kairós. 